\title{
L’impact de la mobilité linguistique sur l'évolution démographique des francophones du Québec
} THE IMPACT OF LINGUISTIC MOBILITY ON THE DEMOGRAPHIC EVOLUTION OF QUEBEC FRANCOPHONES IMPACTO DE LA MOVILIDAD LINGUISTICA SOBRE LA EVOLUCION DEMOGRAFICA DE LA POBLACION DE HABLA FRANCESA DE QUEBEC

\section{Marc Termote}

Volume 27, numéro 2, automne 1998

Malthus

URI : https://id.erudit.org/iderudit/010252ar

DOI : https://doi.org/10.7202/010252ar

Aller au sommaire du numéro

Éditeur(s)

Association des démographes du Québec

ISSN

0380-1721 (imprimé)

1705-1495 (numérique)

Découvrir la revue

Citer cet article

Termote, M. (1998). L'impact de la mobilité linguistique sur l'évolution démographique des francophones du Québec. Cahiers québécois de démographie, 27(2), 267-294. https://doi.org/10.7202/010252ar

\section{Résumé de l'article}

L'objectif de cet article est de vérifier dans quelle mesure une mobilité linguistique croissante vers le français serait capable de freiner, voire de renverser, les effets du manque de dynamisme démographique du groupe francophone du Québec. Pour répondre a cette question, un grand nombre d'hypothèses d'évolution future du comportement linguistique, toutes très favorables au groupe francophone, ont été combinées à divers contextes démographiques. Les résultats de tous les scénarios convergent vers la même conclusion : l'impact de la mobilité linguistique est marginal, de telle sorte que la décroissance de l'effectif et du poids du groupe francophone semble inéluctable. Cette conclusion vaut particulièrement pour l'île de Montréal, où le déclin de l'effectif francophone est déja amorcé et où les francophones deviendront minoritaires dans une dizaine d'années. 
Cahiers québécois de démographie

Vol. 27, no 2, automne 1998, p. 267-294.

\title{
L'impact de la mobilité linguistique sur l'évolution démographique des francophones du Québec
}

\author{
Marc TERMOTE *
}

Depuis plusieurs décennies, le comportement démographique des francophones du Québec est tel que la quasi-totalité des prévisions aboutissent à la conclusion selon laquelle la décroissance de ce groupe, en termes tant d'effectifs que de part relative, semble, sinon inéluctable, du moins très difficile à éviter. En effet, la conjonction d'une sous-fécondité chronique (surtout sur lîle de Montréal, où la fécondité des francophones est même inférieure à celle des anglophones), d'une immigration internationale très majoritairement non francophone, et d'une migration interne qui ne procure aucun gain au groupe francophone (sauf autour de Montréal, où l'étalement urbain joue de façon marquée contre le groupe francophone de l'ile) ne peut que conduire à une décroissance démographique.

Dans ces conditions, on peut ètre tenté de trouver dans la mobilité linguistique une solution permettant de contrer la tendance fondamentale vers la décroissance du groupe franco-

Institut national de la recherche scientifique (INRS-Urbanisation), Université du Québec, 3465, rue Durocher, Montréal, et Centre interuniversitaire d'êtudes démographiques (CIED), Montréal. L'auteur tient à remercier Jacques Ledent, qui a développé le modèle de projection démographique multirégionale de manière à y intégrer la mobilité linguistique, et qui a appliqué ce modèle à ses données et à ses scênarios. Il exprime également sa gratitude au Conseil de la langue française et au Ministère des Relations avec les citoyens et de I'Immigration (MRCI), pour toute l'aide obtenue, ainsi qu'à Charles Castonguay et à trois évaluateurs anonymes, pour leurs commentaires et critiques particulièrement riches et constructifs. Il importe de souligner qu'au moment de la rédaction de la version finale de cet article (printemps 1998), les résultats du recensement de 1996 en matière linguistique n'étaient pas encore connus. La comparaison de ces résultats avec ceux du recensement de 1991 est d'ailleurs sujette à caution. Courriel : marc_termote@inrs-urb.uquebec.ca 
phone. En termes plus "politiques", puisqu'une intervention discriminatoire dans le domaine de la fécondité, de l'immigration internationale et de la migration interne est difficilement concevable (sauf, du moins partiellement, dans le cas de l'immigration internationale), on peut se demander si des mesures linguistiques (comme la loi 101) visant à favoriser la force d'attraction du français auprès des membres des groupes non francophones représentent un outil efficace pour lutter contre la tendance à la décroissance démographique manifestée par le groupe francophone. L'objectif de cet article est précisément de vérifier dans quelle mesure une mobilité linguistique croissante vers le français serait capable de freiner, voire de renverser, les effets du manque de dynamisme démographique du groupe francophone.

Pour répondre à cette question, nous avons intégré dans nos dernières perspectives démolinguistiques une multiplicité d'hypothèses de comportement linguistique'. Dans une première étape, nous exposerons comment nous avons élaboré ces hypothèses. Puisque l'impact de la mobilité linguistique sur l'évolution de l'effectif des divers groupes linguistiques dépend aussi du contexte démographique, nous examinerons ensuite brièvement les hypothèses de fécondité, de mortalité et de migration utilisées. Dans la dernière section de cet article, nous analyserons les résultats obtenus.

\section{ESTIMATION ET PRÉVISION DE LA MOBILITÉ LINGUISTIQUE}

Pour pouvoir estimer l'impact de la mobilité linguistique sur l'évolution future de l'effectif des divers groupes linguistiques (définis selon la langue d'usage, c'est-à-dire la langue le plus fréquemment utilisée au sein du ménage), il nous faut disposer pour chaque période (quinquennale, en l'occurrence) de la prévision, d'une estimation des probabilités de passage d'un groupe linguistique à chaque autre, et cela par âge et, dans notre cas, également par région. Or, la seule information disponible concerne la mobilité dite "durée de vie". Ce que l'on appelle communément "transfert linguistique " ne porte en effet que sur un type particulier de mobilité, celui qui s'effectue

I Pour une analyse détaillée des scénarios et des résultats de ces perspectives démolinguistiques, voir Termote, 1996. Le présent article reprend pour l'essentiel l'analyse développée dans ce dernier ouvrage, mais en y intégrant de nouveaux scénarios et en mettant l'accent sur le rôle de la mobilité linguistique. 
entre la langue maternelle et la langue utilisée au sein du ménage au moment du recensement. Une telle information ne permet pas de connaître le moment auquel a été réalisé le transfert, ni donc l'âge au moment de ce transfert, information qui est cependant cruciale, surtout lorsqu'il s'agit d'estimer la mobilité linguistique des immigrants de langue maternelle autre que française et anglaise, dont on ne peut déterminer s'ils ont effectué un transfert avant ou après leur arrivée au Québec. Ainsi, dans le cas d'un immigrant de langue maternelle arabe arrivé au Québec en 1985, si celui-ci déclare au recensement de 1991 que le français est sa langue d'usage, on ne peut savoir s'il a effectué ce transfert après 1985 ou avant (lors de ses études en France, par exemple).

Une autre implication de ce type de données "durée de vie " est que d'un recensement à l'autre le nombre de transferts vers une langue donnée peut diminuer (augmenter), même si la force d'attraction de cette langue reste identique : il suffit qu'il y ait émigration (immigration) parmi les membres de ce groupe ayant effectué ce transfert. Ainsi, dans le cas du Québec, une forte émigration des anglophones (parmi lesquels il y a certes nombre d'immigrants de langue maternelle "autre ") y fait baisser le nombre de transferts effectués vers l'anglais, de telle sorte que l'on pourrait conclure à une baisse de la force d'attraction de cette langue, même si en réalité cela n'était pas le cas.

Tout comme on ne peut faire une prévision des mouvements migratoires en utilisant des données de migration "durée de vie " (obtenues en comparant le lieu de naissance et le lieu de résidence au moment du recensement), de même on ne peut utiliser directement les données sur les transferts linguistiques "durée de vie". Par contre, sur la base de ces données à deux recensements successifs, on peut tenter (moyennant la réserve exprimée ci-dessus) d'estimer le nombre de transferts réalisés au cours de cette période censitaire qui soit compatible avec le nombre de transferts "durée de vie" observé en fin de période. Une telle estimation a été réalisée pour l'ensemble du Québec pour la période 1981-1986 par Girard (1992) (voir aussi Robitaille et al,, 1992). Le tableau 1 présente les résultats obtenus.

Comme toute estimation, celle-ci est bien sûr toujours sujette à caution. Mais comme d'autres procédures (tout aussi discutables...) produisent des résultats comparables, nous baserons notre argumentation sur les chiffres du tableau 1. Il 
TABLEAU 1 - Estimation du nombre de transferts linguistiques effectués au cours de la période 1981-1986 au Québec

\begin{tabular}{lrcrr}
\hline $\begin{array}{l}\text { Langue d'origine } \\
\text { (utilisée } \\
\text { en 1981) }\end{array}$ & Français & Anglais & Autre & Total \\
\cline { 2 - 5 } & - & 13442 & 0 & 13442 \\
Français & 9964 & - & 0 & 9964 \\
Anglais & 3394 & 6889 & - & 10283 \\
Autre & 13358 & 20331 & 0 & 33689 \\
Total & -84 & 10367 & -10283 & 0 \\
Solde & &
\end{tabular}

Source : Girard, 1992 (voir aussi Robitaille et al., 1992 : 153).

ressort de ce tableau que le nombre de transferts linguistiques effectués au cours d'une période quinquennale est très faible : pour l'ensemble du Québec, on obtient un grand total de moins de 34000 transferts (toutes langues "d'origine " et toutes langues de "destination " confondues), soit moins de 7000 transferts par an. Un tel résultat ne devrait guère surprendre, car un individu ne change pas aisément de langue d'usage. Un transfert linguistique ne se fait que dans des circonstances exceptionnelles et après un processus d'" assimilation "particulièrement long, s'étendant souvent sur plusieurs générations.

On peut raisonnablement supposer que la très grande majoritè des transferts ont lieu entre 20 et 40 ans (comme on le verra plus loin, les transferts effectués avant l'âge de 20 ans, essentiellement liés à la langue utilisée à l'école, ne peuvent être considérés comme définitifs). Connaissant, grâce à une compilation spéciale du recensement de 1991, le nombre de transferts "durée de vie " pour chaque région et pour chaque paire origine-destination, nous avons utilisé l'image quinquennale présentée dans le tableau 1 pour obtenir des probabilités de transferts entre chaque groupe linguistique, et cela par groupe quinquennal d'âge et région. Les probabilités estimées pour 1981-1986 ont donc servi de base à l'élaboration des hypothèses de mobilité linguistique utilisées dans le cadre de notre étude, étant entendu que nous n'avons pas repris ces probabilités telles quelles, car on peut espérer que depuis 1986-1991, grâce en partie au fait que la loi 101 a commencé à exercer ses pleins effets, la force d'attraction du français a augmenté, surtout auprès des allophones.

Le passage des données "durée de vie "à des estimations et des prévisions par période quinquennale implique que toute 
hypothèse de mobilité linguistique est nécessairement fragile. Selon que l'on est optimiste ou pessimiste quant à la capacité des individus de changer de langue d'usage et quant à la force d'attraction d'une langue par rapport à une autre, et selon que l'on est optimiste ou pessimiste quant à l'efficacité des mesures gouvernementales visant à influencer cette capacité individuelle, on jugera qu'il y a ici un risque de biais. Heureusement, le nombre de transferts au cours d'une période quinquennale est tellement faible par rapport au nombre de naissances, décès et migrations, que l'impact d'une erreur, même considérable, dans l'estimation et la prévision des probabilités de transfert est relativement marginal. C'est d'ailleurs ce qui ressortait déjà de notre étude prévisionnelle précédente (Termote, 1994), ainsi que de l'étude de Robitaille et al. (1992) mentionnée plus haut.

Un des principes de base de toute prévision est de ne pas multiplier le nombre d'hypothèses pour les facteurs qui n'exercent qu'un rôle secondaire dans l'évolution du phénomène à prévoir. Dans notre cas, nous avons dérogé à cette règle, puisqu'il s'agissait ici d'estimer l'impact d'un facteur (la mobilité linguistique) dont la mesure (les probabilités de transfert sur une période quinquennale) est particulièrement fragile. Aussi, afin d'éviter que ne soient remis en cause nos résultats sous prétexte d'une mauvaise estimation du comportement linguistique présent et futur, non seulement nous avons multiplié le nombre d'hypothèses, mais nous les avons systématiquement - et parfois outrancièrement - biaisées en faveur du français. De ce point de vue, tous nos résultats surestiment sans aucun doute nettement le poids des francophones, du moins à court et moyen terme.

Outre l'hypothèse de mobilité linguistique nulle (MLO), qui n'est évidemment pas une hypothèse prévisionnelle, mais qui permet d'estimer directement l'impact de cette mobilité, nous avons retenu cinq hypothèses. Avant d'expliciter (par âge, région et groupe linguistique) les probabilités quinquennales de transfert adoptées, il importe de dégager brièvement la structure fondamentale de ce que pourrait ètre le comporternent linguistique de la population québécoise à court et moyen terme.

Une première caractéristique sur laquelle la plupart des études s'entendent est que la mobilité linguistique n'est guère significative avant 20 ans. Sans doute des transferts (relativement peu nombreux, d'ailleurs) sont-ils déclarés (le plus souvent par un tiers, c'est-à-dire le "chef " de famille) pour des jeunes de moins de 20 ans, mais de tels transferts peuvent 
difficilement être considérés comme définitifs, car dans la majorité des cas ils sont liés à la langue utilisée à l'école, langue qui pour la plupart de ces jeunes transferts (ceux des "enfants de la loi 101 ") est la langue "obligatoire ". Dès que ce caractère obligatoire est levé, on observe d'ailleurs un changement dans le choix de la langue d'enseignement, comme en témoignent les résultats d'une enquête récente de la Commission des écoles catholiques de Montreal (CECM, 1996), selon laquelle un tiers des allophones inscrits en dernière année d'une école secondaire francophone ont déclaré poursuivre leurs études collégiales dans un établissement anglophone. C'est à la sortie du réseau scolaire que l'on peut juger de la signification d'un transfert linguistique. Le contexte linguistique du milieu de travail et celui du ménage (en relation donc avec le comportement "matrimonial ", selon qu'il y a endogamie ou exogamie) sont beaucoup plus déterminants, dans la mesure où ils vont en quelque sorte "fixer" un éventuel transfert préparé par l'école. C'est pourquoi nous supposerons qu'il n'y a pas de mobilité linguistique avant 20 ans. De même, nous ferons l'hypothèse qu'il n'y en a pas après 40 ans, ce que les études sur les transferts "duree de vie "font clairement apparaitre (Termote et Gauvreau, 1988 : 138-142); plus on avance en âge, plus il est difficile de changer de langue d'usage.

Une deuxième caractéristique de nos hypothèses de mobilité linguistique réside dans le postulat qu'aucun transfert ne se fait vers les langues " autres ". Sans doute cette hypothèse estelle irréaliste, mais le nombre de transferts "durée de vie " de ce type est tellement faible qu'il est fort probable qu'il n'y en ait que quelques centaines par période quinquennale pour l'ensemble du Québec.

La troisième caractéristique de nos hypothèses est que la propension des francophones à changer de langue d'usage est très faible et décroissante (cette hypothèse de décroissance n'est sans doute guère valable pour le passé, mais elle vise comme bien d'autres - à " biaiser " notre analyse en faveur du groupe francophone), alors que nous supposons que celle des anglophones augmente significativement. Selon le recensement de 1991 , à peine $3,7 \%$ des membres du groupe de langue maternelle française résidant sur lîle de Montréal avaient effectué un transfert en termes de durée de vie. Nous avons supposé que dorénavant ils sont encore moins portés à abandonner leur langue, et nous leur avons attribué dès 1991 des probabilités de transfert qui impliquent en termes de durée de vie un total 
de 2,7 transferts pour 100 personnes, soit une réduction de plus de $25 \%$ par rapport à ce qui a été observé en 1991. Par contre, toujours dans le souci de maximiser la chance de voir apparaitre pour le groupe francophone un impact positif suffisamment élevé pour être significatif, nous avons supposé que les probabilités de passage de l'anglais vers le français doublent, ce qui implique un total de 13,5 transferts "durée de vie " pour 100 membres du groupe anglophone au lieu de six. anterrieurement.

Il reste finalement à estimer les probabilités cle passage au français et à l'anglais parmi les membres du groupe "autre ". $\grave{A}$ cet effet, il nous faut au préalable faire une hypothèse sur le moment auquel ont eu lieu les transferts "durée de vie" déclarés lors du recensement. Dans le cas des immigrants de ce groupe, on ne peut savoir si le transfert a eu lieu avant ou après l'arrivée au Québec. Mais puisque l'âge moyen des immigrants au moment de leur immigration est relativement élevé (proche de la trentaine), il est fort probable que peu d'entre eux feront un transfert après leur arrivée au Québec, puisque les données "durée de vie "montrent que la quasitotalité des transferts ont lieu avant 40 ans, surtout entre 20 et 30 ans. Il faut cependant également tenir compte des natifs de langue "autre ", qui eux peuvent - par définition - effectuer un transfert au Québec tout au long de leur vie. C'est pourquoi, même si les immigrants de langue "autre " représentent plus de la moitié des membres du groupe "autre ", nous avons supposé que parmi l'ensemble des membres de ce groupe le pourcentage de transferts réalisés au Québec s'élève à $80 \%$. Il s'agit fort probablement d'une très forte surestimation, mais celle-ci nous permet à nouveau de donner au groupe francophone le maximum de chances de faire des gains par mobilité linguistique.

Encore faut-il répartir ces transferts entre ceux qui sont destinés au groupe français et ceux qui sont destinés au groupe anglais. On sait qu'en termes de "durée de vie ", la majorité (selon le recensement de 1991, 63\% pour l'ensemble du Québec, $70 \%$ sur l'ille de Montréal) des transferts ont lieu vers l'anglais (seules les déclarations de langue unique étant prises en compte). Mais on sait aussi que, grâce en partie à la législation linguistique en vigueur depuis 20 ans, la force d'attraction du français par rapport à celle de l'anglais a augmenté, du moins parmi les jeunes immigrants. C'est pourquoi il nous faut distinguer au sein du groupe " autre " quatre sous-groupes. 
TABLEAU 2 - Répartition des personnes natives de langue maternelle autre que française, anglaise et autochtone, selon la langue parlée à la maison, Québec, 1991

\begin{tabular}{|c|c|c|c|c|c|c|c|c|c|}
\hline \multirow{2}{*}{$\begin{array}{l}\text { Groupe } \\
\text { d'âge }\end{array}$} & \multicolumn{2}{|c|}{ Français } & \multicolumn{2}{|c|}{ Anglais } & \multicolumn{2}{|c|}{ Autre } & \multicolumn{2}{|c|}{$\begin{array}{l}\text { Réponses } \\
\text { multiples }\end{array}$} & \multirow{2}{*}{$\begin{array}{c}\text { Total } \\
= \\
100 \%\end{array}$} \\
\hline & $\mathrm{N}$ & $\%$ & $\mathrm{~N}$ & $\%$ & $\mathrm{~N}$ & $\%$ & $\mathrm{~N}$ & $\%$ & \\
\hline Total & 13475 & 9,9 & 52065 & 38,1 & 56645 & 41,5 & 14400 & 10,5 & 136585 \\
\hline $0-14$ & 4455 & 8,3 & 10315 & 19,3 & 32805 & 61,5 & 5795 & 10,9 & 53370 \\
\hline $15-24$ & 2260 & 6,4 & 13620 & 38,6 & 14480 & 41,0 & 4955 & 14,0 & 35315 \\
\hline $25-29$ & 1180 & 7,2 & 9105 & 55,6 & 4655 & 28,4 & 1425 & 8,7 & 16365 \\
\hline $30-44$ & 2405 & 14,0 & 10300 & 59,8 & 3160 & 18,4 & 1350 & 7,8 & 17215 \\
\hline $45-64$ & 1680 & 21,9 & 4710 & 61,3 & 905 & 11,8 & 385 & 5,0 & 7680 \\
\hline $65+$ & 1495 & 22,5 & 4015 & 60,5 & 640 & 9,6 & 490 & 7,4 & 6640 \\
\hline $0-14$ & 4455 & 8,3 & 10315 & 19,3 & 32805 & 61,5 & 5795 & 10,9 & 53370 \\
\hline $15+$ & 9020 & 10,8 & 41750 & 50,2 & 23840 & 28,6 & 8605 & 10,3 & 83215 \\
\hline $25+$ & 6760 & 14,1 & 28130 & 58,7 & 9360 & 19,5 & 3650 & 7,6 & 47900 \\
\hline
\end{tabular}

Source: Recensement 1991, compilation spéciale, Ministère des Relations avec les citoyens et de l'Immigration (MRCI).

Les autochtones (Amérindiens et Inuit) constituent un bloc non négligeable (quelque $10 \%$ du total des allophones) et leur propension à effectuer un transfert vers le français est plutôt faible, et le restera sans doute encore longtemps. Les natifs dont la langue maternelle n'est ni le français, ni l'anglais, ni une langue autochtone, constituent un second sous-groupe, dont le poids dans le total du groupe "autre " est important (quelque $35 \%$ ). Les chiffres (langue unique) du tableau 2 montrent que les membres de ce sous-groupe ont très majoritairement choisi l'anglais lorsqu'ils ont fait un transfert: le rapport est presque de 1 à 4 (9,9\% contre 38,1\%). La force d'attraction de l'anglais auprès des jeunes natifs est particulièrement impressionnante : au sein du groupe des 15-24 ans, près de $40 \%$ ont choisi l'anglais, contre à peine $6 \%$ le français; et parmi les 25-29 ans, plus de 55\% ont adopté l'anglais, contre $7 \%$ le français. On remarquera que parmi les enfants de moins de 15 ans (donc nés après la promulgation de la loi 101), le rapport entre le nombre de transferts effectués vers le français et le nombre de transferts réalisés vers l'anglais est de 1 à 2 , soit à peu près le même rapport que celui observé pour l'ensemble des membres du groupe de langue maternelle " autre ". Dans ces conditions, il semble peu réaliste de compter sur les membres du sous-groupe constitué des natifs pour augmenter significativement la propension globale (de l'ensemble du groupe de langue " autre ") à devenir francophone. 


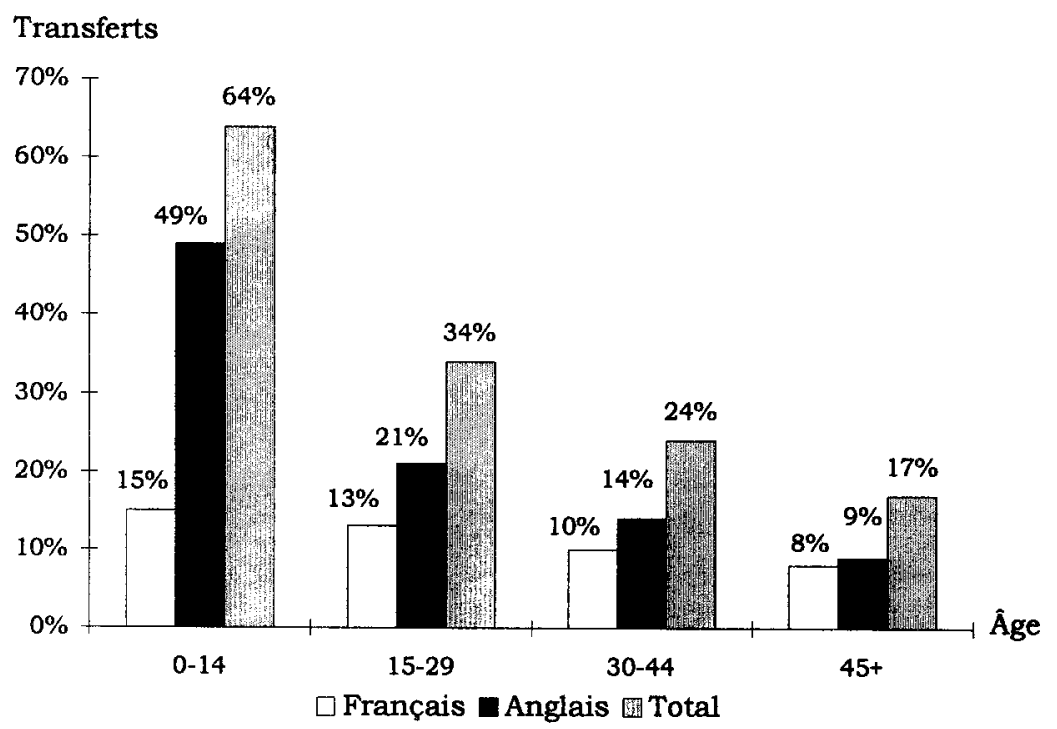

Source : MCCI.

FIGURE 1 - Transferts chez les allophones * immigrés selon l'âge à l'immigration, cohortes avant 1976, Québec, 1991 * langue maternelle unique)

Le troisième sous-groupe est constitué des immigrants de langue maternelle "autre "immigrés avant 1976 (avant la loi 101). La figure 1 montre que pour ce sous-groupe également la préférence pour l'anglais, lorsque transfert il y a (transfert dont on ne sait pas s'il a eu lieu avant ou après l'immigration), est nette, et cela quel que soit l'âge de l'immigrant (âge à l'arrivée, et non au recensement). La seule exception concerne les 45 ans et plus (âge au moment de l'immigration), pour lesquels le français parvient à faire jeu égal (probablement à cause des transferts effectués avant l'arrivée), mais très peu d'immigrants (à peine $17 \%$ ) de ce groupe d'âge ont effectué un transfert. On remarquera la forte prédominance de l'anglais parmi ceux qui sont arrivés avant l'âge de 15 ans $(15 \%$ ont choisi le français, contre près de $50 \%$ l'anglais) : cela traduit bien l'efficacité " assimilatrice " de l'école anglaise à l'époque du libre choix de la langue scolaire.

Il ne reste finalement que le sous-groupe constitué par les immigrés de langue maternelle autre arrivés après 1976 qui témoigne d'une préférence pour le français en cas de transfert. La figure 2 donne une image d'ensemble, dont il ressort que pour le total des immigrés de la période 1976-1991, et pour 


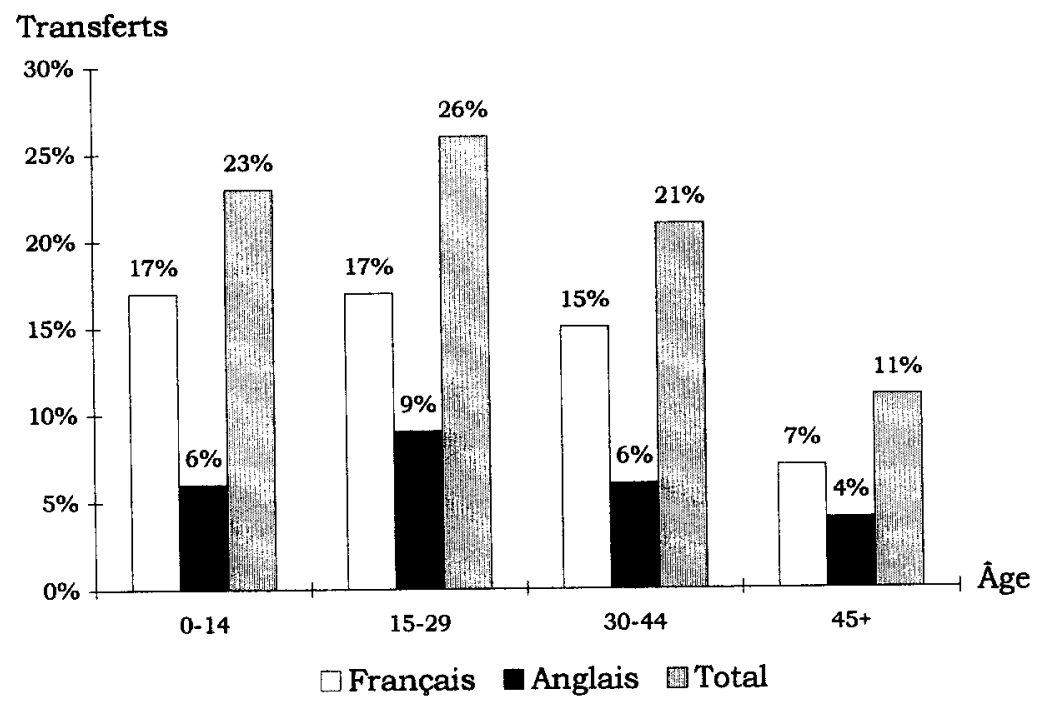

Source : MRCI.

FIGURE 2 - Transferts chez les allophones * immigrés selon l'âge à l'immigration, cohortes 1976-1991, Québec, 1991 (* langue maternelle unique)

tout le Québec, le rapport est de 1 à 3 en faveur du français parmi les 0-14 ans, et de 1 à 2 parmi les 15-29 ans. Il faut cependant remarquer que ces rapports ne portent que sur un nombre relativement faible de transferts (respectivement $23 \%$ et $26 \%$ ont effectué un transfert, et le pourcentage est encore beaucoup moins élevé pour les autres groupes d'âge).

En outre, et surtout, il importe de souligner que la situation semble avoir évolué récemment d'une manière moins favorable au français. Le tableau 3 présente - par groupe quinquennal d'âge - les taux de transfert linguistique parmi les immigrants de langue maternelle "autre" (langue unique) arrivés entre 1986 et 1991, dernière période connue. Comme la plupart de ces immigrants se sont êtablis sur l'île de Montréal, et que c'est dans cette région que l'incidence des transferts linguistiques est la plus importante, nous nous limiterons à celle-ci. Les chiffres de ce tableau montrent que parmi les immigrants récents il y a quasiment égalité entre la force d'attraction du français et celle de l'anglais (sauf pour les moins de 20 ans, pour lesquels le rapport est de 1 à 2 en faveur du français). Sans doute faut-il tenir compte de la durée d'exposition au " risque " : la plupart des immigrants de 1986-1991 n'ont pas 
TABLEAU 3 - Taux de transfert linguistique par âge, parmi les immigrants de langue maternelle autre (langue unique) arrivés entre 1986 et 1991, ile de Montréal

\begin{tabular}{lcccc}
\hline $\begin{array}{l}\text { Age en } \\
1991\end{array}$ & Français & Anglais & Autre & Multiples \\
\hline $5-9$ & 8,5 & 4,2 & 77,3 & 10,0 \\
$10-14$ & 9,2 & 4,2 & 76,1 & 10,5 \\
$15-19$ & 7,7 & 4,9 & 77,0 & 10,4 \\
$20-24$ & 9,0 & 9,1 & 72,5 & 9,4 \\
$25-29$ & 10,7 & 11,2 & 69,1 & 9,0 \\
$30-34$ & 8,8 & 10,0 & 70,8 & 10,4 \\
$35-39$ & 8,9 & 10,5 & 71,1 & 9,5 \\
$40-44$ & 8,8 & 8,3 & 74,4 & 8,5 \\
$45-49$ & 7,6 & 7,7 & 76,9 & 7,8 \\
$50-54$ & 6,6 & 5,1 & 81,1 & 7,2 \\
$55-59$ & 6,8 & 4,7 & 81,6 & 6,9 \\
$60-64$ & 3,8 & 4,8 & 85,3 & 6,1 \\
$65-69$ & 3,5 & 3,5 & 85,2 & 7,8 \\
$70-74$ & 4,4 & 4,4 & 83,9 & 7,3 \\
$75-79$ & 4,6 & 4,6 & 86,2 & 4,6 \\
$80-84$ & 1,7 & 1,7 & 93,2 & 3,4 \\
$85+$ & 3,3 & 3,3 & 86,7 & 6,7 \\
Total & 8,6 & 8,1 & 74,0 & 9,3 \\
\hline
\end{tabular}

Source : Statistique Canada, compilations spéciales du recensement de 1991 et calculs de l'auteur.

encore eu le temps d'effectuer un transfert. Il faut cependant remarquer que cela était vrai également pour les immigrants des périodes antérieures. Or, à partir du recensement de 1981 on avait pu observer (Termote et Gauvreau, 1988: 153) une prédominance du français très nette parmi les immigrants de langue maternelle " autre " arrivés entre 1976 et 1981, soumis à la même durée d'exposition au risque. Il y a donc bien eu détérioration entre 1976-1981 et 1986-1991. En fait, comme l'ont démontré Baillargeon et Benjamin (1990) et Castonguay (1994, 1997), cette détérioration avait déjà commencé à se manifester entre 1981 et 1986.

Enfin, il faut remarquer que les taux de transfert très favorables (en termes relatifs, c'est-à-dire par rapport à l'anglais) observés pour le français parmi les jeunes immigrants de 19861991 portent sur des petits nombres. Pour les moins de 15 ans, cela concerne moins de 1500 personnes, soit seulement $11 \%$ des transferts effectués par l'ensemble des immigrants arrivés 
entre 1986 et 1991 , et à peine $1 \%$ du total des transferts réalisés par l'ensemble des membres de langue " autre " résidant en 1991 sur l'île de Montréal. On peut d'ailleurs se demander ce que signifie un transfert à cet âge, surtout si les parents (les 25-39 ans) des enfants de cet âge ont choisi majoritairement l'anglais. En outre, ce sont ces mêmes parents qui sont les auteurs de la déclaration linguistique de leurs enfants...

Dans ces conditions, il semble difficile de soutenir que la moitié des transferts de l'ensemble des membres du groupe "autre " se font ou se feront à court et moyen terme vers le français, en se fondant sur le fait qu'en 1991 la majorité des quelques milliers de jeunes immigrants récents qui ont fait un transfert ont déclaré (eux ou leurs parents) avoir choisi le français comme langue d'usage. On ne peut extrapoler le comportement de quelques milliers de personnes à tout un groupe (celui de l'ensemble des personnes de langue maternelle " autre ") qui en comporte 400000.

C'est cependant en toute connaissance de cause que, dans une première étape, nous ferons cette hypothèse d'égalité entre la force d'attraction du français et celle de l'anglais pour l'ensemble des membres du groupe de langue maternelle "autre ", même si à court et moyen terme une telle hypothèse semble peu réaliste. Encore une fois, nous avons fait ce type d'hypothèse pour maximiser les chances de voir apparaitre un facteur favorable à l'évolution démographique future du groupe francophone. Le tableau 4 présente les probabilités de transfert que nous avons utilisées pour chaque groupe linguistique et pour chaque groupe quinquennal d'âge (entre 20 et 40 ans). Nous avons supposé que ces probabilités pouvaient s'appliquer à chacune des trois régions du Québec considérées dans notre analyse (l'̂̀le de Montréal, le reste de la région métropolitaine de Montréal et le reste du Québec), ce qui favorise sans nul doute le groupe francophone de l'ile de Montréal. Puisque ces diverses probabilités quinquennales surestiment nettement la force d'attraction du français, du moins à court et moyen terme, nous les avons maintenues constantes tout au long de la période de prévision. Enfin, pour pouvoir "fermer " le système, il nous fallait estimer également des probabilités de transfert par période quinquennale pour le "Canada moins Québec "; ces probabilités sont présentées dans la seconde partie du tableau 4. Les diverses probabilités présentées dans ce tableau constituent notre première hypothèse de mobilité linguistique, notre hypothèse de base (ML1). 
TABLEAU 4-Hypothèse ML1 de mobilité linguistique. Probabilités quinquennales de transfert (en \%) entre groupes linguistiques par groupe d'âge, Québec et "Canada moins Québec ", 1991-2021 (taux constants)

A. Pour chaque région du Québec

\begin{tabular}{lcccc}
\hline \multicolumn{2}{l}{ Àge et langue d'origine } & Français & $\begin{array}{c}\text { Langue d'usage } \\
\text { Anglais }\end{array}$ & Autre \\
\hline $20-24$ ans & F & - & 7,6 & 0,0 \\
& A & 40,0 & - & 0,0 \\
$25-29$ ans & $\varnothing$ & 42,0 & 42,0 & - \\
& F & - & 7,2 & 0,0 \\
$30-34$ ans & A & 38,0 & - & 0,0 \\
& F & 38,0 & 38,0 & - \\
$35-39$ ans & A & - & 6,6 & 0,0 \\
& Ø & 34,0 & - & 0,0 \\
& F & - & 34,0 & - \\
& A & 30,0 & 6,2 & 0,0 \\
& Ø & 30,0 & - & 0,0 \\
\hline
\end{tabular}

B. "Canada moins Québec"

\begin{tabular}{lcccc}
\hline Âge et langue d'origine & Français & $\begin{array}{c}\text { Langue d'usage } \\
\text { Anglais }\end{array}$ & Autre \\
\hline \multirow{2}{*}{$20-24$ ans } & F & - & 40,0 & 0,0 \\
& A & 0,0 & - & 0,0 \\
$25-29$ ans & $\varnothing$ & 0,0 & 84,0 & - \\
& F & - & 38,0 & 0,0 \\
$30-34$ ans & A & 0,0 & - & 0,0 \\
& F & 0,0 & 76,0 & - \\
$35-39$ ans & A & 0,0 & - & 0,0 \\
& $\varnothing$ & 0,0 & 68,0 & 0,0 \\
& F & - & 30,0 & 0,0 \\
& A & 0,0 & - & 0,0 \\
\hline
\end{tabular}

NOTES

1. Pour le Québec, ces probabilités impliquent 2,7 transferts durée de vie pour 100 membres du groupe de langue maternelle française, 13,5 transferts pour $100 \mathrm{du}$ groupe anglais et 27 transferts pour $100 \mathrm{du}$ groupe " autre " (13,5 vers le français et 13,5 vers l'anglais).

2. $\mathrm{F}=$ français; $\mathrm{A}=$ anglais; $\varnothing=$ autre. 
Non content d'avoir ainsi introduit une multiplicité de biais en faveur du groupe francophone, particulièrement celui de l'ile de Montréal, nous avons cependant voulu aller jusqu'au bout de l'argumentation qui vise à attribuer à ce groupe le maximum de chances de regagner par mobilité linguistique ce qu'il perd par ailleurs. Aussi avons-nous supposé, dans une seconde hypothèse (ML2), que les probabilités du tableau 4 (partie A), même si elles surestiment considérablement la force d'attraction actuelle du français, vont évoluer de manière à accroître encore cette force d'attraction. Plus précisément, nous avons supposé que progressivement les probabilités de passage du groupe "autre " vers le français augmenteront de façon à ce que dans un quart de siècle ce dernier devienne aussi attractif pour l'ensemble des allophones du Québec que l'anglais ne l'est actuellement pour les allophones du reste du Canada. Cette hypothèse de mobilité linguistique croissante vers le français implique que le nombre de transferts durée de vie (55 au total) effectués par les membres du groupe "autre " du Québec sera alors relativement proche de celui actuellement observé parmi les membres de ce groupe résidant dans le reste du Canada. Le tableau 5 explicite ces diverses probabilités.

TABLEAU 5 - Hypothèse ML2 de mobilité linguistique. Probabilités quinquennales de mobilité linguistique (en \%o) entre groupes linguistiques par groupe d'âge, Québec et "Canada moins Québec ", 1991-2021 a

Toutes les probabilités sont identiques à celles de l'hypothèse ML1 (tableau 4), sauf les quatre probabilités de transfert du groupe " autre "vers le français au Québec : pour chacune des régions du Québec, ces probabilités sont augmentées de $20 \%$ (soit $2 \%$ en points de pourcentage) à chaque lustre, à partir de 2001-2006 jusqu'à 2016-2021; pour 1991-1996 on applique les probabilités du tableau 4 et pour 1996-2001 ces probabilités sont augmentées de $10 \%$. Nous avons donc les probabilités (en pour mille) suivantes pour les transferts du groupe "autre" au groupe francophone :

\begin{tabular}{lllllll}
\hline Âge & $1991-$ & $1996-$ & $2001-$ & $2006-$ & $\begin{array}{l}2011- \\
2016\end{array}$ & $\begin{array}{l}2016- \\
2021\end{array}$ \\
\hline $20-24$ & 1996 & 2001 & 2006 & 2011 & 2012,0 \\
$25-29$ & 42,0 & 52,0 & 72,0 & 92,0 & 112,0 & 132,0 \\
$30-34$ & 38,0 & 48,0 & 68,0 & 88,0 & 108,0 & 128,0 \\
$35-39$ & 34,0 & 44,0 & 64,0 & 84,0 & 104,0 & 124,0 \\
\hline
\end{tabular}

a. Ces probabilités impliquent que le nombre total de transferts " durée de vie " pour 100 personnes du groupe "autre " passe de 27 (dont la moitié vers le français) en 1991-1996 à 55,3 en 2016-2021 (dont 41,7, soit $75 \%$, vers le français). Rappelons qu'il s'agit de transferts réalisés au Québec (et non avant leur arrivêe lorsqu'il s'agit d'immigrants). 
TABLEAU 6 - Hypothèse ML3 de mobilité linguistique. Probabilités quinquennales de transfert (en \%o) entre groupes linguistiques par groupe d'âge, Québec et "Canada moins Québec ", 1991-2021 (taux constants) a

Toutes les probabilités du "Canada moins Québec "sont les raêmes que celles de l'hypothèse ML1, mais pour chaque région du Québec on applique dès 1991-1996 les probabilitês suivantes :

\begin{tabular}{|c|c|c|c|c|}
\hline \multirow{2}{*}{\multicolumn{2}{|c|}{ Âge et langue d'origine }} & \multicolumn{3}{|c|}{ Langue d'usage } \\
\hline & & Français & Anglais & Autre \\
\hline \multirow[t]{3}{*}{$10-14$ ans } & $\mathrm{F}$ & - & 0,0 & 0,0 \\
\hline & A & 0,0 & - & 0,0 \\
\hline & $\varnothing$ & 500,0 & 250,0 & - \\
\hline \multirow[t]{3}{*}{$15-19$ ans } & $\mathrm{F}$ & - & 0,0 & 0,0 \\
\hline & $A$ & 0,0 & - & 0,0 \\
\hline & $\varnothing$ & 20,0 & 20,0 & - \\
\hline \multirow{3}{*}{$20-24$ ans } & $\mathbf{F}$ & - & 7,6 & 0,0 \\
\hline & A & 40,0 & - & 0,0 \\
\hline & $\varnothing$ & 20,0 & 20,0 & - \\
\hline \multirow[t]{3}{*}{$25-29$ ans } & $F$ & - & 7,2 & 0,0 \\
\hline & A & 38,0 & - & 0,0 \\
\hline & $\varnothing$ & 20,0 & 20,0 & - \\
\hline \multirow[t]{3}{*}{ 30-34 ans } & $\mathrm{F}$ & - & 6,6 & 0,0 \\
\hline & A & 34,0 & - & 0,0 \\
\hline & $\varnothing$ & 20,0 & 20,0 & - \\
\hline \multirow[t]{3}{*}{$35-39$ ans } & $\mathrm{F}$ & - & 6,2 & 0,0 \\
\hline & $A$ & 30,0 & - & 0,0 \\
\hline & $\varnothing$ & 20,0 & 20,0 & - \\
\hline
\end{tabular}

a. Pour le Québec, ces probabilités impliquent que le nombre de transferts "durée de vie " pour 100 personnes du groupe " autre " s'élèverait à 80 , dont 53 vers le français et 27 vers l'anglais, le nombre de transferts des deux autres groupes étant identique à celui supposé dans le scénario ML1 (tableau 4).

Puisque toute estimation de la mobilité linguistique par période est nécessairement fragile, il est évidemment très facile de remettre en cause des hypothèses basées sur une telle estimation, et d'avancer que les deux hypothèses précédentes ne sont pas encore assez favorables au groupe francophone. C'est pourquoi nous avons introduit une hypothèse extrême (appelée ML3), selon laquelle dès 1991 les enfants du groupe de langue " autre " âgés de 10-14 ans réalisent pour les trois quarts un transfert linguistique définitif, et cela dans une proportion de deux transferts vers le français pour un transfert vers l'anglais. Après cet âge, les rares personnes de ce groupe qui n'ont pas encore effectué de transfert sont soumises à des probabilités quinquennales de transfert relativement faibles $(2 \%$ dans les deux cas). Le tableau 6 présente les diverses probabilités de transfert utilisées dans le cadre de cette hypothèse ML3 de très 
forte mobilitê immédiate vers le français. Une telle hypothèse revient à attribuer dès 1991 à l'ensemble de la population de langue " autre "le comportement que l'on peut espérer observer parmi les enfants de la seconde génération d'immigrants soumis à la loi 101. Elle implique que le nombre de transferts "durée de vie " par 100 personnes de langue maternelle " autre " s'élèverait au Québec à 80 , dont 53 vers le français et 27 vers l'anglais, ce qui témoignerait d'une mobilité linguistique nettement supérieure à celle observée dans le "reste du Canada ", alors qu'actuellement c'est l'inverse qui prévaut.

Comme une telle "surmobilité "linguistique de la part des allophones du Québec est totalement irréaliste, nous avons tempéré quelque peu le caractère extrême de cette hypothèse en introduisant une quatrième hypothèse (ML4), selon laquelle les probabilités de transfert des 10-14 ans sont (dans chaque région du Québec) réduites de moitié par rapport à celles utilisées dans 1'hypothèse ML3, toutes les autres probabilités restant identiques. Cette nouvelle hypothèse implique un total de 53 transferts "durée de vie "pour 100 personnes de langue maternelle " autre ", soit 32 vers le français et 21 vers l'anglais.

L'hypothèse ML3 cumulait une très forte mobilité linguistique immédiate avec une nette prédominance du français sur l'anglais. On pourrait se demander ce qu'il adviendrait si le français et l'anglais exerçaient la même force d'attraction, tout en supposant toujours une très forte mobilité immédiate de la part des allophones. En fait, pour obtenir un nombre de transferts "durée de vie " vers le français relativement proche de celui supposé dans l'hypothèse ML3, nous avons encore augmenté la mobilité linguistique de ces derniers, de telle sorte que le nombre de transferts " durée de vie " des allophones s'élève à 91 pour 100 personnes (46 vers le français et 45 vers l'anglais). En même temps, nous avons encore réduit (de 2,7 à 2,3 transferts par 100 personnes) les probabilités qu'un francophone fasse un transfert vers l'anglais, et augmenté les probabilités (de 13,5 à 18,5 transferts par 100 personnes) qu'un anglophone effectue un transfert vers le français. Les probabilités par âge qui conduisent à cette situation de très forte mobilité linguistique immédiate sans nette prédominance du français auprès des allophones, sont présentées dans le tableau 7 , et constituent l'hypothèse ML5. 
TABLEAU 7 - Hypothèse ML5 de mobilité linguistique. Probabilités quinquennales de transfert (en \%o) entre groupes linguistiques par groupe d'âge, Québec et "Canada moins Québec ", 1991-2021 (taux constants) a

Toutes les probabilités du "Canada moins Québec " sont les mêmes que celles de 1'hypothèse ML1, mais pour chaque région du Québec on applique dès 1991-1996 les probabilitês suivantes

\begin{tabular}{lcccc}
\hline Âge et langue d'origine & Français & $\begin{array}{c}\text { Langue d'usage } \\
\text { Anglais }\end{array}$ & Autre \\
\hline $20-24$ ans & F & - & 6,4 & 0,0 \\
& A & 56,0 & - & 0,0 \\
$25-29$ ans & $\varnothing$ & 265,0 & 260,0 & - \\
& F & - & 6,0 & 0,0 \\
$30-34$ ans & A & 52,0 & - & 0,0 \\
& F & 240,0 & 235,0 & - \\
$35-39$ ans & A & - & 5,6 & 0,0 \\
& $\varnothing$ & 215,0 & - & 0,0 \\
& F & - & 210,0 & 0,0 \\
& $\varnothing$ & 44,0 & 5,2 & 0,0 \\
\hline
\end{tabular}

a. Pour le Québec, ces probabilités impliquent 2,3 transferts " durée de vie " pour 100 membres du groupe français (contre 2,7 selon l'hypothèse ML1), 18,5 transferts pour 100 membres du groupe anglais (contre 13,5), et 91 transferts pour 100 membres du groupe "autre " (contre 27 selon ML1), dont 46 vers le français et 45 vers l'anglais.

\section{HYPOTHĖSES DE COMPORTEMENT DÉMOGRAPHIQUE}

Il est évident que l'impact que peut exercer la mobilité linguistique sur l'évolution de l'effectif des groupes linguistiques ne dépend pas seulement de la mobilité entre ces groupes, mais aussi du comportement de fécondité, de mortalité et de migration adoptê par les membres de ces groupes linguistiques. Par souci de brièveté, il ne nous est pas possible d'expliciter ici les diverses hypothèses utilisées à cet égard. Une présentation succincte devrait cependant permettre d'interpréter de façon appropriée les résultats obtenus (une analyse plus détaillée des hypothèses démographiques est fournie dans Termote, 1996 : 8-19 et 27-33).

Tous les scénarios discutés dans cet article comportent la même hypothèse de mortalité, soit celle d'une baisse progressivement ralentie des taux de mortalité par âge des francophones et des anglophones, les taux observés en 1986-1991 des allophones étant supposés constants, parce que manifestement sous-estimés (les données relatives au nombre de décès selon 
l'âge et la langue d'usage impliquent une espérance de vie des allophones de Montréal supérieure de dix ans à celle de leurs concitoyens francophones et de cinq ans supérieure à celle de leurs concitoyens anglophones). Cette hypothèse fait croître de quelque cinq ans l'espérance de vie des francophones et des anglophones, et cela sur une période de 40 ans. En 2036-2041, cette espérance de vie (sexes confondus) atteindrait 79,4 ans chez les premiers et 84,5 ans chez les seconds, et se maintiendrait à 84,0 ans chez les allophones.

Quatre hypothèses de fécondité ont été utilisées : le maintien des taux de fécondité par âge observés en 1986-1990; le maintien des taux de fécondité estimés pour 1991-1995, ce qui implique une baisse par rapport au comportement de 19861990; la poursuite de la baisse de la fécondité, jusqu'à un indice synthétique "moyen " (pour l'ensemble du Québec, tous groupes linguistiques confondus) de 1,4 en 2001 (en 1996, l'indice estimé par le Bureau de la statistique du Québec s'élève à 1,58 ), avec constance des taux de fécondité par la suite; et une remontée rapide de la fécondité dès 1996-2001, pour atteindre dès 2001 le niveau $(1,8)$ observé en 1986-1990. Cette "fourchette" 1,4-1,8 nous semble représenter, du moins à court et moyen terme, l'éventail le plus plausible. Pour chacune de ces hypothèses, les disparités entre groupes linguistiques, les écarts régionaux et le profil par âge observés en 1986-1990 sont supposés demeurer constants.

Deux hypothèses ont été retenues pour la migration interprovinciale : le maintien (pour chaque groupe linguistique) du comportement migratoire observé en 1986-1991 (ce qui implique un solde annuel négatif de quelque 6000 personnes), et une légère hausse (de $10 \%$ ) de tous les taux d'émigration du Québec vers le reste du Canada couplée à une baisse de $10 \%$ des taux d'émigration de cette dernière région vers le Québec, ce qui aboutirait dès 1991-1996 à une augmentation de la perte migratoire, qui passerait de 6000 personnes à 11000 , solde négatif qui demeurerait plus ou moins constant par la suite. Pour la migration infraprovinciale, une hypothèse de constance des taux observés pour chaque groupe linguistique en 1986-1991 a également été utilisée, de même qu'une seconde hypothèse visant à réduire fortement et rapidement l'étalement urbain autour de l'île de Montréal. Il est à noter qu'une telle réduction de l'étalement urbain se manifeste même avec l'hypothèse de constance des taux, à cause de la décroissance du nombre d'émigrants potentiels due à l'étalement 
urbain des décennies antérieures, et à cause du vieillissement de la population.

Nous n'avons considéré qu'une seule hypothèse d'émigration internationale (des taux d'émigration constants qui impliquent une sortie annuelle de quelques milliers de personnes), parce qu'il s'agit là d'un phénomène mineur. Par contre, nous avons introduit trois hypothèses d'immigration internationale, avec des niveaux qui s'élèvent respectivement à 26000 , 33000 et 40000 immigrants reçus annuellement.

\section{L'IMPACT DE LA MOBILITÉ LINGUISTIQUE}

Puisque l'impact de la mobilité linguistique dépend du contexte démographique dans lequel cette mobilitë opère, nous avons considéré un grand nombre de scénarios de comportement démographique. Les résultats de chacun des scénarios utilisés ne peuvent évidemment être présentés dans le cadre de cet article. Seules les tendances globales pourront être dégagées. Pour ce faire, nous partirons d'un scénario dit "de base", qui parmi les 27 scénarios retenus (les résultats de 22 d'entre eux sont produits dans Termote, 1996) n'est pas nécessairement le plus plausible, mais qui peut être considéré comme le plus favorable au groupe francophone parmi les divers scénarios les plus plausibles.

Selon ce scénario, le comportement de fécondité observé en 1991-1995 est maintenu constant, la mortalité poursuit sa baisse (mais de façon progressivement ralentie), le comportement migratoire tant interprovincial qu'infraprovincial observé en 1986-1991 demeure constant, et pour chaque période quinquennale le nombre annuel moyen d'entrées internationales survivantes en fin de période s'élève à 30000 (ce qui implique quelque 33000 immigrants reçus). Êtant donné l'impact majeur qu'exercent la fécondité et l'immigration internationale sur l'évolution du groupe francophone, particulièrement à Montréal, il semble opportun de nous attarder quelque peu sur l'hypothèse relative à ces deux composantes.

Du moins à court terme, lhypothèse de fécondité retenue dans ce scénario de base ne semble pas devoir se vérifier, car l'on sait que depuis 1995 la fécondité est à nouveau en baisse au Québec. Notre hypothèse implique donc que cette baisse ne serait que temporaire et que la fécondité devrait remonter bientôt, de telle sorte que la moyenne quinquennale de l'indice de fécondité soit proche de celle observée en 1991-1995. Même 
si une telle hypothèse risque donc d'être invalidée, nous l'avons malgré tout retenue dans notre scénario de base, parce qu'elle permet d'introduire un biais supplémentaire en faveur du groupe francophone. Quant à l'hypothèse d'un niveau " moyen " d'immigration internationale, elle semble fort plausible, du moins à court et moyen terme. On sait en effet que si annuellement l'immigration internationale peut fortement varier, la moyenne annuelle calculée sur une période censitaire est beaucoup plus stable. La moyenne annuelle de la période censitaire 1991-1996 sera fort probablement proche de celle supposée dans notre scénario de base, puisque selon les derniers chiffres disponibles, la moyenne annuelle de la période quinquennale 1991-1995 (années civiles) serait de quelque 33000 immigrants reçus.

Il reste à choisir, parmi les cinq hypothèses de mobilité linguistique définies précédemment, celle qui serait la plus plausible. Rappelons que certaines de ces hypothèses ne sont en fait pas des hypothèses prévisionnelles. Toutes les hypothèses caractérisées par une très forte surmobilité $(80 \%$ à $90 \%$ de transferts "durée de vie " chez les allophones et par une très forte attraction immédiate pour le français, sont totalement irréalistes. Il s'agit là d'hypothèses de simulation, destinées à évaluer en quelque sorte par l'absurde l'impact de la mobilité linguistique. L'hypothèse de mobilité linguistique retenue est l'hypothèse ML1, selon laquelle dès 1991 la moitié des transferts effectués par l'ensemble des allophones se font vers le français. Comme une telle hypothèse est irréaliste à court et moyen terme, nous avons supposé la constance des probabilités de transfert qui y sont utilisées.

Le tableau 8 présente les résultats obtenus à la fois avec ce scénario de base et avec un scénario qui reproduit toutes les hypothèses du scénario de base sauf pour la mobilité linguistique, supposée nulle. Cette comparaison entre ce que l'on peut attendre avec l'hypothèse la plus plausible (quoique surestimant l'attraction du français auprès des allophones) et ce qui pourrait se produire avec une hypothèse d'absence de mobilité nous semble la meilleure façon d'estimer l'impact de cette mobilité. Les résultats produits dans ce tableau montrent clairement l'impact marginal exercé par la mobilité linguistique sur l'évolution de la structure linguistique. La part de chaque groupe dans la population totale n'est guère influencée par la mobilité linguistique, particulièrement la part du groupe francophone. Cette conclusion est valable quelle que soit la région 
TABLEAU 8 -Évolution prévisible des effectifs et de la structure linguistique (1) avec mobilité linguistique (scénario de base) et (2) sans mobilité linguistique, 1991-2021

\begin{tabular}{|c|c|c|c|c|c|}
\hline & & $\begin{array}{l}\text { Population } \\
\text { totale }(000)\end{array}$ & $\begin{array}{c}\text { \% franco- } \\
\text { phones }\end{array}$ & $\begin{array}{l}\% \text { anglo- } \\
\text { phones }\end{array}$ & $\begin{array}{l}\% \text { allo- } \\
\text { phones }\end{array}$ \\
\hline \multicolumn{6}{|c|}{ n̂e de Montréal } \\
\hline 1991 & (OBS) & 1776 & 57,3 & 26,2 & 16,5 \\
\hline \multirow[t]{2}{*}{2001} & (1) & 1835 & 52,4 & 25,6 & 22,0 \\
\hline & (2) & 1838 & 51,8 & 25,4 & 22,8 \\
\hline \multirow[t]{2}{*}{2011} & (1) & 1911 & 48,8 & 24,9 & 26,3 \\
\hline & (2) & 1921 & 47,7 & 24,3 & 28,0 \\
\hline \multirow[t]{2}{*}{2021} & (1) & 1976 & 45,8 & 24,3 & 29,9 \\
\hline & (2) & 1995 & 44,2 & 23,3 & 32,5 \\
\hline \multicolumn{6}{|c|}{ Reste R. M. Montréal } \\
\hline 1991 & (OBS) & 1358 & 84,8 & 10,6 & 4,6 \\
\hline \multirow[t]{2}{*}{2001} & (1) & 1620 & 82,6 & 10,5 & 6,9 \\
\hline & (2) & 1619 & 82,5 & 10,3 & 7,2 \\
\hline \multirow{2}{*}{2011} & (1) & 1800 & 80,6 & 10,4 & 9,0 \\
\hline & (2) & 1800 & 80,3 & 9,9 & 9,8 \\
\hline \multirow[t]{2}{*}{2021} & (1) & 1913 & 78,5 & 10,3 & 11,2 \\
\hline & (2) & 1916 & 77,8 & 9,6 & 12,6 \\
\hline \multicolumn{6}{|c|}{ Reste Québec } \\
\hline 1991 & (OBS) & 3762 & 94,3 & 4,4 & 1,3 \\
\hline \multirow[t]{2}{*}{2001} & (1) & 3913 & 93,9 & 4,4 & 1,7 \\
\hline & (2) & 3917 & 94,1 & 4,2 & 1,7 \\
\hline \multirow[t]{2}{*}{2011} & (1) & 4006 & 93,5 & 4,5 & 2,0 \\
\hline & (2) & 4017 & 93,7 & 4,1 & 2,2 \\
\hline \multirow[t]{2}{*}{2021} & (1) & 4015 & 92,9 & 4,7 & 2,4 \\
\hline & (2) & 4034 & 93,1 & 4,2 & 2,7 \\
\hline \multicolumn{6}{|c|}{ Total Québec } \\
\hline 1991 & (OBS) & 6896 & 82,9 & 11,2 & 5,9 \\
\hline \multirow[t]{2}{*}{2001} & $(1)$ & 7367 & 81,1 & 11,0 & 7,9 \\
\hline & $(2)$ & 7375 & 81,0 & 10,8 & 8,2 \\
\hline \multirow[t]{2}{*}{2011} & (1) & 7717 & 79,4 & 10,9 & 9,7 \\
\hline & (2) & 7738 & 79,2 & 10,5 & 10,3 \\
\hline \multirow[t]{2}{*}{2021} & (1) & 7905 & 77,6 & 11,0 & 11,4 \\
\hline & (2) & 7945 & 77,1 & 10,3 & 12,8 \\
\hline
\end{tabular}

considérée. Même en longue période, après 30 ans, l'écart entre le poids du groupe francophone avec et sans mobilité linguistique n'est que de $1,6 \%$ en points de pourcentage lorsqu'il s'agit de lîle de Montréal (la CUM), qui est cependant la région 
où - comme on pouvait s'y attendre - l'impact de la mobilité linguistique est le plus élevé.

En ce qui concerne l'évolution prévisible du poids des divers groupes linguistiques, on peut observer que le groupe francophone deviendra minoritaire sur l'île de Montréal peu après l'an 2001 , et qu'entre 2001 et 2011 le poids des allophones y sera supérieur à celui des anglophones. Cette double conclusion est valable qu'il y ait ou non mobilité linguistique. On notera également que le poids des francophones diminuera dans toutes les rëgions.

Pour vérifier la robustesse de ces conclusions, on peut adopter une approche beaucoup plus extrême, en quelque sorte "par l'absurde ", qui consiste à supposer un comportement de mobilité linguistique totalement irréaliste, où par exemple les allophones manifestent dès 1991 une très forte surmobilité linguistique $(80$, voire 90 transferts "durée de vie " pour 100 personnes, un nombre qui est le double de ce qui est observé aujourd'hui au Québec et qui est $50 \%$ plus élevé que celui observé dans le reste du Canada). Si même ce type d'hypothèse n'invalide pas les conclusions dégagées précédemment, on pourra vraiment clore le débat relatif à la capacité de la mobilité linguistique de renverser les tendances démographiques défavorables du groupe francophone. Le tableau 9 présente l'évolution prévisible du poids des francophones selon les diverses hypothèses de mobilité linguistique discutées précédemment.

Les résultats présentés dans ce tableau montrent clairement que la prise en considération de la mobilité linguistique - même la plus extrême $(91$ transferts durée de vie pour 100 allophones : hypothèse ML5) - conduit à des résultats relativement peu différents de ceux obtenus par une hypothèse de mobilité nulle. Cela est valable même pour l'île de Montréal, où cependant - à cause de la forte présence des allophones l'impact de la mobilité linguistique est le plus élevé. Dans cette dernière région, après 15 ans (entre 1991 et 2006) l'écart entre les deux hypothèses extrêmes (MLO et ML5) n'est que de 3,6\% en points de pourcentage, soit une variation de $7 \%$. Même après 30 ans, l'écart ne s'élève qu'à $6,7 \%$ en points de pourcentage.

Les conclusions relatives à la minorisation du groupe francophone sur líle de Montréal et à la baisse du poids de ce groupe dans toutes les régions restent également valables. Bien sûr, plus on augmente la mobilité linguistique des allophones 
TABLEAU 9 - Pourcentage de francophones selon diverses hypothèses de mobilité linguistique

\begin{tabular}{lcccccc}
\hline & MLO & ML1 & ML2 & ML3 & ML4 & ML5 \\
\hline \multicolumn{7}{c}{ Montréal (CUM) } \\
\hline 1991 & 57,3 & 57,3 & 57,3 & 57,3 & 57,3 & 57,3 \\
2006 & 49,7 & 50,5 & 50,8 & 51,7 & 51,0 & 53,3 \\
2021 & 44,2 & 45,8 & 47,2 & 48,5 & 47,0 & 50,9 \\
\hline \multicolumn{7}{c}{ Reste de la région métropolitaine } \\
\hline 1991 & 84,8 & 84,8 & 84,8 & 84,8 & 84,8 & 84,8 \\
2006 & 81,4 & 81,6 & 81,7 & 82,3 & 81,9 & 83,2 \\
2021 & 77,8 & 78,5 & 79,4 & 80,5 & 79,4 & 82,2 \\
\hline \multicolumn{7}{c}{ Reste du Québec } \\
\hline 1991 & 94,3 & 94,3 & 94,3 & 94,3 & 94,3 & 94,3 \\
2006 & 93,9 & 93,7 & 93,7 & 93,9 & 93,8 & 94,1 \\
2021 & 93,1 & 92,9 & 93,1 & 93,4 & 93,1 & 93,8 \\
\hline
\end{tabular}

NOTE

MLO : Mobilité linguistique nulle.

$\mathrm{ML1}: \mathrm{F} \rightarrow \mathrm{A}: 2,7 ; \mathrm{A} \rightarrow \mathrm{F}: 13,5 ; \varnothing \rightarrow \mathrm{F}: 13,5 ; \varnothing \rightarrow \mathrm{A}: 13,5$

ML2 : idem sauf $\emptyset \rightarrow \mathrm{F}: 41,7 ; \emptyset \rightarrow \mathrm{A}: 13,6$ en $2016-2021$

ML3 : idem sauf $\varnothing \rightarrow F: 53,0 ; \emptyset \rightarrow A: 27,0$ dès 1991

ML4 : idem sauf $\emptyset \rightarrow F: 32,0 ; \emptyset \rightarrow A: 21,0$ dès 1991

ML5 : $\mathrm{F} \rightarrow \mathrm{A}: 2,3 ; \mathrm{A} \rightarrow \mathrm{F}: 18,5 ; \varnothing \rightarrow \mathrm{F}: 46 ; \varnothing \rightarrow \mathrm{A}: 45$ dès 1991

Où $F=$ français; $A=$ anglais; $\emptyset=$ autre, et où les chiffres indiquent le nombre de transferts " durée de vie "(pour 100 personnes) impliqué par les probabilités quinquennales de transfert (par âge) utilisées dans l'hypothèse.

et les probabilités de transfert vers le français, plus on freine le déclin du poids du groupe francophone et plus on recule les échéances. Mais ces gains hypothétiques ne sont guère impressionnants: même les hypothèses les plus irréalistes (ML3, MLA, et ML5) ne font reculer que de 5 à 15 ans le moment où les francophones deviendront minoritaires sur lîle de Montréal.

Derrière tous ces pourcentages, derrière l'évolution prévisible de la part de chaque groupe linguistique, il y a les nombres. À bien des égards, l'évolution des effectifs est au moins aussi importante que celle des pourcentages. Une dernière manière de vérifier le caractère marginal de la mobilité linguistique en ce qui concerne l'évolution démolinguistique du Québec est de déterminer la période au cours de laquelle débu- 
TABLEAU 10 - Période quinquennale au cours de laquelle débutera le déclin de l'effectif des francophones, selon la région et le scénario

\begin{tabular}{cccccc}
\hline $\begin{array}{l}\text { No du } \\
\text { scé- } \\
\text { nario }\end{array}$ & $\begin{array}{c}\text { Île de } \\
\text { Mont- } \\
\text { réal }\end{array}$ & $\begin{array}{c}\text { Reste de } \\
\text { la région } \\
\text { métro }\end{array}$ & $\begin{array}{c}\text { Total } \\
\text { région } \\
\text { métro }\end{array}$ & $\begin{array}{c}\text { Reste } \\
\text { du } \\
\text { Québec }\end{array}$ & $\begin{array}{c}\text { Total } \\
\text { du } \\
\text { Québec }\end{array}$ \\
\hline 1 & $1991-1996$ & $2026-2031$ & $2016-2021$ & $2016-2021$ & $2016-2021$ \\
2 & $1991-1996$ & $2026-2031$ & $2021-2026$ & $2016-2021$ & $2016-2021$ \\
3 & $1991-1996$ & $2031-2036$ & $2031-2036$ & $2016-2021$ & $2021-2026$ \\
4 & $1991-1996$ & $2031-2036$ & $2031-2036$ & $2016-2021$ & $2021-2026$ \\
5 & $1991-1996$ & $2031-2036$ & $2026-2031$ & $2016-2021$ & $2016-2021$ \\
6 & $1991-1996$ & $2026-2031$ & $2016-2021$ & $2011-2016$ & $2016-2021$ \\
7 & $1991-1996$ & $2031-2036$ & $2026-2031$ & $2016-2021$ & $2016-2021$ \\
8 & $1991-1996$ & $2031-2036$ & $2026-2031$ & $2016-2021$ & $2016-2021$ \\
9 & $1991-1996$ & $2031-2036$ & $2031-2036$ & $2016-2021$ & $2021-2026$ \\
10 & $1991-1996$ & $2026-2031$ & $2021-2026$ & $2016-2021$ & $2016-2021$ \\
11 & $1991-1996$ & $2031-2036$ & $2031-2036$ & $2016-2021$ & $2021-2026$ \\
12 & $1991-1996$ & $2031-2036$ & $2026-2031$ & $2016-2021$ & $2016-2021$ \\
13 & $1991-1996$ & $2031-2036$ & $2031-2036$ & $2016-2021$ & $2021-2026$ \\
14 & $1991-1996$ & $2021-2026$ & $2016-2021$ & $2016-2021$ & $2016-2021$ \\
15 & $1991-1996$ & $2026-2031$ & $2016-2021$ & $2016-2021$ & $2016-2021$ \\
16 & $1991-1996$ & $2031-2036$ & $2026-2031$ & $2016-2021$ & $2016-2021$ \\
17 & $1991-1996$ & $2031-2036$ & $2026-2031$ & $2016-2021$ & $2016-2021$ \\
18 & $1991-1996$ & $2026-2031$ & $2021-2026$ & $2016-2021$ & $2016-2021$ \\
19 & $1991-1996$ & $2026-2031$ & $2021-2026$ & $2016-2021$ & $2016-2021$ \\
20 & $1991-1996$ & $2031-2036$ & $2031-2036$ & $2016-2021$ & $2021-2026$ \\
21 & $1991-1996$ & $2021-2026$ & $2021-2026$ & $2016-2021$ & $2016-2021$ \\
22 & $1991-1996$ & $2026-2031$ & $2031-2036$ & $2016-2021$ & $2021-2016$ \\
\hline
\end{tabular}

Source : Termote, 1996 : tableau 38.

tera la diminution de l'effectif des francophones selon les divers scénarios. Le tableau 10 présente ce type de résultat. À cause de son caractère extrême, nous en avons exclu l'hypothèse de mobilité linguistique ML5. Mais, contrairement aux tableaux précédents, qui supposaient toujours un même comportement démographique "de base ", le tableau 10 présente une grande variété de scénarios (22 au total), que nous avons inclus afin de vérifier si un autre contexte de fécondité et (ou) de migration (tant internationale qu'interprovinciale ou infraprovinciale) pouvait modifier significativement les conclusions dégagées précédemment $^{2}$.

Selon l'information présentée dans le tableau 10, la décroissance de l'effectif des francophones a déjà commencé sur lîle

2 L'explicitation des hypothèses utilisées dans chacun de ces 22 scénarios n'est évidemment pas possible dans le cadre de cet article. Le lecteur intéressé trouvera une analyse détaillée de ces scênarios dans Termote, $1996: 24-43$ et tableau 15. 
de Montréal ${ }^{3}$, et cela quelle que soit l'hypothèse de mobilité linguistique (le comportement démographique estimé pour 1991-1996 est le même dans tous les scénarios). Une modification future du comportement démographique ne renverse pas cette tendance déjà amorcée. Dans le reste de la région métropolitaine de Montréal, qui bénéficie d'une surfécondité francophone (toute relative) et de l'étalement urbain (très majoritairement francophone), et où l'immigration internationale (très majoritairement non francophone) n'exerce guère d'influence, l'échéance du déclin est beaucoup plus éloignée, mais on ne peut manquer de souligner la remarquable convergence des résultats : quel que soit le contexte démographique, qu'il y ait hausse ou baisse de la fécondité, qu'il y ait peu ou beaucoup d'immigrants internationaux, qu'il y ait étalement urbain important ou non, on se retrouve toujours dans la même "fourchette " 2026-2036.

La même convergence se manifeste en ce qui concerne le reste du Québec, et ce de façon encore plus nette : tous les scénarios mènent à une décroissance des effectifs débutant en 2016-2021 (sauf un seul - d'ailleurs proche de l'évolution actuelle - impliquant une poursuite temporaire de la baisse de la féconditê, ce qui a pour effet d'anticiper d'un lustre le début du déclin). Au total, pour l'ensemble du Québec, la baisse de l'effectif francophone devrait commencer dans une vingtaine d'années, ou au mieux un lustre plus tard, ce qui ne fait qu'expliciter en termes démolinguistiques les résultats globaux obtenus par le Bureau de la statistique du Québec, selon lesquels la décroissance de la population totale du Québec devrait s'amorcer dans une quarantaine d'années. Le poids croissant de la population allophone, qui bénéficie d'un dynamisme clémographique remarquable (fécondité assurant le renouvellement " naturel ", faible émigration, forte immigration internationale) permet en effet de reculer quelque peu l'échéance du déclin pour l'ensemble de la population québécoise.

\section{CONCLUSIONS}

L'impressionnante convergence des résultats obtenus à partir d'un grand nombre de scénarios, qui couvrent un large éventail des comportements de fécondité, de migration et de

3 Les résultats préliminaires du recensement de 1996 confirment d'ailleurs cette prévision : l'effectif francophone de l'île de Montréal serait passé de 1018 mille en 1991 à 973 mille en 1996. 
mortalité les plus plausibles, avec des hypothèses de mobilité linguistique allant de l'optimisme (la moitié des transferts de l'ensemble des allophones étant supposés être effectués dès 1991 vers le français) à l'utopie (80\% à $90 \%$ de transferts "durée de vie " parmi les allophones, avec très forte attraction pour le français), montre bien la robustesse de nos conclusions.

Celles-ci peuvent être résumées en deux points. Le premier concerne l'influence secondaire, voire marginale, de la mobilité linguistique sur l'évolution démolinguistique de la société québécoise, même lorsque cette mobilité se présente sous sa forme la plus utopiquement favorable au français. Une telle conclusion peut sembler surprenante si l'on considère que nos hypothèses impliquent toutes que presque tous les allophones ou leurs descendants de la première ou de la seconde génération finiront par être "absorbés" par le groupe francophone. Il importe à cet égard de distinguer les effets "micro ", liês au comportement individuel, des effets " macro", relatifs à l'action des facteurs autres que la mobilité linguistique. En effet, pendant tout le temps que prend le processus d'assimilation linguistique d'un individu non francophone au groupe francophone, d'autres phénomènes, comme la sous-fécondité des francophones, l'êtalement urbain autour de lîle de Montrêal, l'immigration internationale majoritairement non francophone, continuent d'exercer un impact négatif majeur sur le poids et l'effectif du groupe francophone. Les gains que ce dernier peut espérer réaliser par la mobilité linguistique ne pourront jamais compenser l'effet négatif des facteurs démographiques. Une telle conclusion rejoint celle qu'avaient dégagée Robitaille et al. (1992) à partir d'une autre approche (et dans un cadre plus restreint). Le fait que deux études indépendantes, menées selon des méthodologies différentes, aboutissent à la même conclusion renforce évidemment la robustesse de celle-ci.

La seconde conclusion, qui dérive directement de la première, concerne l'apparente inéluctabilité du déclin de l'effectif et de la part du groupe francophone, et ce dans chacune des trois régions considérées (lî̀le de Montréal, le reste de la région métropolitaine et le reste du Québec). Une telle conclusion peut sembler "pessimiste". Il faut cependant remarquer qu'elle a été obtenue en supposant une augmentation dans certains cas tout à fait irréaliste de la force d'attraction $d u$ français auprès des membres des groupes anglophone et, surtout, allophone. Si le pouvoir d'attraction du français devait demeurer au niveau actuel, et a fortiori s'il 
devait diminuer, on aboutirait à une évolution encore plus défavorable au groupe francophone. Or, un tel scénario est loin d'être exclu, car lorsqu'un groupe linguistique voit décroitre sa part relative, et surtout lorsqu'il devient minoritaire (comme cela est le cas sur 1'île de Montréal), il est fort probable que les membres des autres groupes seront de moins en moins enclins à le rejoindre.

Il importe de souligner que notre analyse a porté sur la langue utilisée au sein du ménage, et cela en quelque sorte par définition, puisqu'il s'agissait d'estimer les effets de la mobilité linguistique. On peut évidemment discuter de la pertinence de ce concept, et avancer que la langue utilisée au travail ou pendant les loisirs, celle adoptée avec les amis, ou encore celle à laquelle il est fait recours pour les relations avec l'autorité publique, peut représenter une catégorie tout aussi significative. Une telle approche permet sans doute d'évacuer les problèmes et les défis qu'impliquent les résultats obtenus en termes de langue d'usage au foyer, mais elle néglige une dimension fondamentale, à savoir que la langue d'usage au sein d'un ménage devient normalement la langue maternelle des enfants de ce ménage. Dans une perspective générationnelle, donc de long terme, dans une société qui a une mémoire et qui entend transmettre son patrimoine identitaire, la langue d'usage " au foyer " semble un concept incontournable.

\section{RÉFÉRENCES BIBLIOGRAPHIQUES}

BAILLARGEON, M., et C. BENJAMIN, 1990. Caractéristiques linguistiques de la population immigrée au Québec en 1986. Montréal, Ministère des Communautés culturelles et de l'Immigration.

CASTONGUAY, C., 1994. L'assimilation linguistique: mesure et évolution 1971-1986. Québec, Conseil de la langue française, $243 \mathrm{p}$.

CASTONGUAY, C., 1997. "Évolution de l'assimilation linguistique au Québec et au Canada entre 1971 et 1991 ", Recherches sociographiques, XXXVIII, $3:$ 469-490.

CECM, 1996. Résultats d'un sondage effectué en juin 1996 auprès des finissants des écoles secondaires françaises de la CECM. Montréal, CECM, $29 \mathrm{p}$.

GIRARD, C. 1992. Développement d'un modèle de projection et analyse de la sensibilité de la composition linguistique de la population du Québec. Montréal, Université de Montréal, Département de démographie, mémoire de maitrise, 93 p. et annexes. 
ROBITAILLE, N., et al., 1992. "L'avenir des groupes linguistiques au Quêbec : équilibres et options ", Cahiers québécois de démographie, $21,2: 147-160$.

TERMOTE, M., 1994. L'Avenir démolinguistique du Québec et de ses régions. Québec, Conseil de la langue française, $266 \mathrm{p}$.

TERMOTE, M., 1996. "Perspectives démolinguistiques du Québec et de la région de Montréal, 1991-2041", publié en annexe dans Le français, langue commune. Enjeu de la société québécoise. Rapport du comité interministériel sur la situation de la langue française. Québec, Les Publications du Québec, 75 p. [plus 39 tableaux et 2 graphiques].

TERMOTE, M., et D. GAUVREAU, 1988. La Situation démolinguistique du Québec. Quêbec, Conseil de la langue française, $282 \mathrm{p}$. 